\title{
Pediatric acquired demyelinating syndromes: a nationwide validation study of the Danish National Patient Register
}

This article was published in the following Dove Press journal:

Clinical Epidemiology

\author{
Magnus Spangsberg Boesen' \\ Melinda Magyari2,3 \\ Alfred Peter Born' \\ Lau Caspar Thygesen ${ }^{4}$ \\ 'Department of Pediatrics, \\ Rigshospitalet, Copenhagen \\ University Hospital, Copenhagen, \\ Denmark; ${ }^{2}$ The Danish Multiple \\ Sclerosis Registry, Department of \\ Neurology, Rigshospitalet, University \\ of Copenhagen, Denmark; ${ }^{3}$ Danish \\ Multiple Sclerosis Center, Department \\ of Neurology, Rigshospitalet, \\ Copenhagen University Hospital, \\ Copenhagen, Denmark; ${ }^{4}$ National \\ Institute of Public Health, University \\ of Southern Denmark, Copenhagen, \\ Denmark
}

Objective: To validate the Danish National Patient Register's (NPR) diagnoses of pediatric acquired demyelinating syndromes (ADS) including multiple sclerosis (MS).

Study design and setting: We identified ADS diagnostic groups using International Classification of Diseases (ICD) codes and reviewed medical records to validate the NPR diagnoses during 2008-2015.

Results: Among 409 children in the study, 184 children had a validated and final ADS diagnosis after reviewing medical records as follows: optic neuritis (ON; $n=46)$, transverse myelitis (TM; $\mathrm{n}=16$ ), acute disseminated encephalomyelitis (ADEM; $\mathrm{n}=50$ ), clinically isolated syndrome (CIS) including dissemination in space (CIS [DIS]) but not dissemination in time $(n=6)$, neuromyelitis optica spectrum disorder (NMOsd; $n=5)$, and MS $(n=61)$. During the mean follow-up of 4.6 years, $33 \%$ of children initially diagnosed with monophasic ADS progressed to MS. Positive predictive value (PPV) was 0.71 (95\% confidence interval [CI] $=0.62-0.80)$ for $\mathrm{ON}, 0.64(95 \% \mathrm{CI}=0.43-0.82)$ for $\mathrm{TM}, 0.93(95 \% \mathrm{CI}=0.84-0.98)$ for $\mathrm{MS}$, $0.27(95 \% \mathrm{CI}=0.19-0.35)$ for CIS, $0.43(95 \% \mathrm{CI}=0.10-0.82)$ for NMOsd, and $0.15(95 \%$ $\mathrm{CI}=0.10-0.20)$ for ADEM. Assuming complete coverage for non-MS ADS, the sensitivity was $0.99(95 \% \mathrm{CI}=0.93-1.00)$ for $\mathrm{ON}, 0.83(95 \% \mathrm{CI}=0.36-1.00)$ for CIS (DIS), and 0.80 $(95 \% \mathrm{CI}=0.56-0.94)$ for $\mathrm{TM}$, but only $0.58(95 \% \mathrm{CI}=0.43-0.72)$ for ADEM and $0.60(95 \%$ $\mathrm{CI}=0.15-0.95)$ for NMOsd.

Conclusion: PPV was high for MS and considered acceptable for ON and TM; therefore, these ICD revision 10 (ICD-10) codes from the NPR are useful for epidemiological studies. Conversely, PPV was low for CIS and ADEM; NMOsd was inconclusive.

Keywords: pediatric MS, acquired demyelinating syndrome, ADEM, health registers, validation, ICD-10

\section{Introduction}

The Danish National Patient Register (NPR) is a nationwide register that has routinely collected administrative and health-related data on all hospital admissions in Denmark since $1977 .{ }^{1}$ Despite numerous validation studies of the NPR, ${ }^{2-13}$ the validity of the International Classification of Diseases revision 10 (ICD-10) codes for pediatric acquired demyelinating syndromes (ADS) has not been examined. The NPR may provide easily accessible population-based disease surveillance and facilitate epidemiological research in pediatric ADS.

However, challenges exist unique to interpreting diagnostic ICD-10 codes for ADS:
Correspondence: Magnus Spangsberg Boesen

Department of Pediatrics, Rigshospitalet, Copenhagen University Hospital, Blegdamsvej 9, 2100 Copenhagen, Denmark

Tel +452763 4945

Email magnus.spangsberg.boesen@ regionh.dk 
1. Presumed monophasic ADS (eg, optic neuritis [ON]) can progress to multiple sclerosis (MS) or neuromyelitis optica spectrum disorder (NMOsd), causing the monophasic ADS to represent the onset of MS or NMOsd at a later point. Therefore, the true incidence of monophasic ADS decreases as follow-up increases if relapses of monophasic ADS convert the child's diagnosis to MS or NMOsd; accordingly, NMOsd and MS could be underdiagnosed with a short follow-up due to reporting delay. ${ }^{14,15}$

2. Once a person is diagnosed with MS, a new clinical event with ON may be interpreted in light of MS and therefore be coded as a relapse of MS instead of ON.

3. Difficulty in comparing the diagnoses of MS and NMOsd over time is reflected by continuous revisions of the diagnostic criteria for MS with the inclusion of the McDonald criteria in 2001, facilitating the use of magnetic resonance imaging (MRI) to show dissemination in space and time, and, for NMOsd with the incorporation of aquaporin-4 (AQ4) antibodies in 2006. ${ }^{16-20}$ However, the diagnostic ICD-10 codes for MS (G35) and NMOsd (G36.0) have not changed in line with these revisions, causing misclassification of patient groups over time.

4. Discrepancy exists between the proposed consensus criteria for acute disseminated encephalomyelitis (ADEM) and how pediatric neurologists diagnose ADEM in clinical practice, particularly regarding whether encephalopathy and polyfocal neurological deficits are required for ADEM..$^{21,22}$

5. Requirements in published articles vary for disease progression to MS in children initially diagnosed with ADEM because some researchers diagnose MS after any relapse, whereas the criteria of the International Pediatric MS Study Group (IPMSSG) state that a second clinical event must be without encephalopathy and an MRI demonstrating dissemination in space. ${ }^{21,23}$

6. Neurologists in Denmark may be more inclined to code a child with $\mathrm{ON}$ as "demyelinating disease of central nervous system (CNS), unspecified" (G37.9) instead of ON (H46) if the child also has dissemination in space on the MRI and cerebrospinal fluid oligoclonal band positivity.

Using the validation study reporting guidelines proposed by Benchimol et al, ${ }^{24}$ we aimed to estimate common epidemiological outcome measures, such as the positive predictive value (PPV) and sensitivity of diagnostic ICD-10 codes, for pediatric ADS in the NPR during 2008-2015, using retrospective chart review as the gold standard.

\section{Methods}

\section{The Danish NPR}

The NPR was established in 1977 and contains nationwide information on all patients admitted to Danish hospitals. Data include date of admission and final diagnoses according to the ICD-8 (1977-1993) and ICD-10 (1994 until today). ${ }^{1}$ At hospital discharge, physicians code each patient by diagnosis with one primary diagnosis and, if relevant, one or more secondary diagnoses. Patients can receive more diagnoses at other hospital discharges or at ambulatory hospital consultations. In Denmark, hospital admissions and outpatient visits are tax funded and free of charge. Patients are coded at each visit, giving chronically diseased patients multiple registrations. ${ }^{2}$ Private consultant physicians (eg, general practitioners, neurologists, and ophthalmologists) and private hospitals in Denmark play a minor role in the diagnostic procedure and always refer children with suspected demyelinating disease to public hospitals.

\section{The Danish Civil Registration System}

The Danish Civil Registration System was established in 1968 as a register of residents in Denmark. ${ }^{13,25}$ In Denmark, every resident is provided with a unique personal identification number that includes date of birth and sex. The personal identification number is used for multiple purposes in Denmark, allowing linkage of patients between various registers. Variables include date of migration and vital status.

\section{Validation cohort}

At onset, all patients were younger than 18 years and living in Denmark. In 2010, Denmark's population was 5,547,683, with $1,196,891$ (22\%) younger than 18 years. ${ }^{26}$ Denmark has a relatively homogeneous Caucasian population.

During January 1, 2008, to December 31, 2015, we identified children in the NPR using non-MS ADS diagnostic codes in the Danish version of ICD-10. Subsequently, we reviewed the children's medical records to verify their diagnoses. We used the following diagnostic ICD-10 codes (ICD-10 in brackets): ON (H46), transverse myelitis (TM) (G37.3), and NMOsd (G36.0). As a specific diagnostic code for demyelinating events caused by supratentorial lesions does not exist, we defined this as "clinically isolated syndrome" (CIS) (G36.8, G36.9, G37.8, and G37.9). Similarly, we defined a diagnostic group for ADEM (G04.0, G04.8, and G04.9). For the latter two groups, ICD-10 codes were defined pre hoc with expertise from both adult and pediatric neurologists based on presumed standards for diagnostic 
coding among physicians. All patients' diagnostic ICD-10 codes of ADS were included (Table S1).

Owing to the risk of developing a chronic demyelinating disease, children with non-MS ADS are usually referred to hospitals as either admissions or outpatient ambulatory consultations, with subsequent ICD-10 code notification to the NPR. Due to the structure of the Danish health care system with few private consultant pediatricians or pediatric ophthalmologists, it is unlikely that children with non-MS ADS are not registered with one of the aforementioned diagnostic codes. In Denmark, patients with ON before the age of 18 years are referred to a hospital for further diagnostic workup.

\section{Definition of diagnoses}

The term ADS refers to all acquired demyelinating diseases of the CNS. ADS can be divided into the following two groups: 1) MS and 2) non-MS ADS, the latter consisting of ON, TM, NMOsd, ADEM, and CIS. CIS included children with ADS who could not be classified into one of the other categories, eg, hemispheric and brainstem demyelinating syndromes; in addition, we defined CIS including dissemination in space but not dissemination in time as CIS (DIS). "Dissemination in space" refers to $\geq 1 \mathrm{~T} 2 \mathrm{MRI}$ lesions in at least two of the four MS typical regions of the CNS (periventricular, juxtacortical, infratentorial, or spinal cord). ${ }^{20}$ "True diagnoses" were based on the chart review including laboratory and MRI investigations. For the chart review, MS was defined by the McDonald criteria at the time of diagnosis, either the 2005 revision or the 2010 revision. ${ }^{19,20}$ NMOsd was defined by the Wingerchuk criteria. ${ }^{17} \mathrm{ON}$ was based on the ophthalmologist's final diagnosis. In Denmark, diagnostic workup of ON includes AQ4 antibody testing with enzyme-linked immunosorbent assay technique if the $\mathrm{ON}$ is bilateral, recidivating or associated with TM. TM was defined as neurological symptoms from the spine verified by a spinal MRI with corresponding lesions and a brain MRI not fulfilling the MS McDonald criteria. ${ }^{20} \mathrm{~A}$ diagnosis of ADEM included an abnormal MRI and neurologic symptoms with a varying degree of encephalopathy and neurological deficits. Progression from ADEM to MS was defined by IPMSSG criteria. ${ }^{21}$ As children with monophasic ADS (such as ON) can progress to relapsing ADS, we recorded whether children initially diagnosed with presumed monophasic ADS progressed to relapsing disease (NMOsd or MS) during follow-up (June 1, 2016).

MSB, who is a medical doctor and has 5 years of clinical training including 1 year of pediatrics and 2 years of neurology, reviewed the medical records under the supervision of a senior pediatric neurologist. MSB was not blinded to children's diagnostic codes during chart review. Case notes were reviewed during June-August 2016.

\section{Statistical methods}

We calculated PPVs including exact confidence intervals (CIs) as the proportion of children in each ICD-10 diagnostic group who truly had the ADS of interest and, based on population numbers in Denmark, the negative predictive values as the proportion of children without a diagnostic code of the ADS of interest who truly did not have the ADS of interest. Sensitivity was estimated as the proportion of children with the specific ICD-10 code among those who truly had the ADS of interest, and specificity was estimated as the proportion of children without the specific ICD-10 code among those who truly did not have the ADS of interest. Likelihood ratios were estimated as the probability of children having the ICD-10 code of ADS of interest who truly had the ADS of interest divided by the probability of the same finding in children who did not truly have the ADS of interest.

We calculated the point prevalence of ADS among children $(<18$ years of age) in Denmark as the sum of the age-specific incidence rates times the remaining years up to 18 years of age by using date of onset (and not date of diagnosis) as onset of ADS. This is appropriate as the rates of migration and death among Danish children and adolescents are low. ${ }^{26}$

We calculated PPVs based on the inclusion of primary diagnoses only, one diagnostic code registration among primary and secondary diagnoses, and at least two code registrations among primary and secondary diagnoses. In addition to calculating the PPV in each diagnostic group for the specific ADS, we calculated the PPV in each group for any ADS. Furthermore, we calculated the proportion of children with presumed monophasic ADS who progressed to relapsing disease (MS, NMOsd or multiphasic ADEM) during follow-up. The statistical analyses were performed using SAS version 9.4 (SAS Institute Inc., Cary, NC, USA).

\section{Ethics}

The study was approved by the Danish Data Protection Agency (case number: 30-1423/03567) and the Danish Health Data Authority (case number: 00001716). The Danish Health and Medicines Authority waived the requirement to obtain patient informed consent to access medical records (case number: 3-3013-896/1). 


\section{Results}

\section{Validation process}

We included 415 children during 2008-2015 using the aforementioned ADS diagnostic codes (Figure 1), giving 1,087 diagnostic ICD-10 code registrations for ADS. Medical records were accessed electronically; none was missing. We excluded four children because they were foreign residents and, therefore, the follow-up case notes were incomplete, and two children because chart review revealed that ADS onset was before the study period. After reviewing the medical records, 184 children truly had ADS, among whom 61 children progressed to MS during follow-up. During follow-up of all 415 children, 13 children died and two children emigrated; among these 15 children, only one child was truly diagnosed with ADS. Among the five children with NMOsd, one child had ON and positive AQ4, one child had ON and subsequently TM but negative AQ4, and three children had TM and positive AQ4; two children with NMOsd were tested for anti-myelin oligodendrocyte glycoprotein (MOG) antibodies, and one test was positive. For the remaining children initially diagnosed with monophasic ADS, we retrieved the following information regarding AQ4 and MOG antibodies (positive tests/total known test results): ADEM: AQ4 (1/11), MOG (1/2); ON: AQ4 (0/8), MOG (1/2); CIS (DIS): AQ4 (0/0), MOG (0/0); and TM: AQ4 (0/9), MOG (1/2).

\section{Baseline characteristics}

For ADEM, the median age at onset was 5.4 years, whereas the median age for the other ADS was 12 years or older at onset. The majority of children with ADEM and TM were boys; for the other ADS groups, girls formed the majority (Table 1).

\section{PPV of ADS}

The PPV in children with at least one diagnostic code as either primary discharge diagnosis or secondary discharge

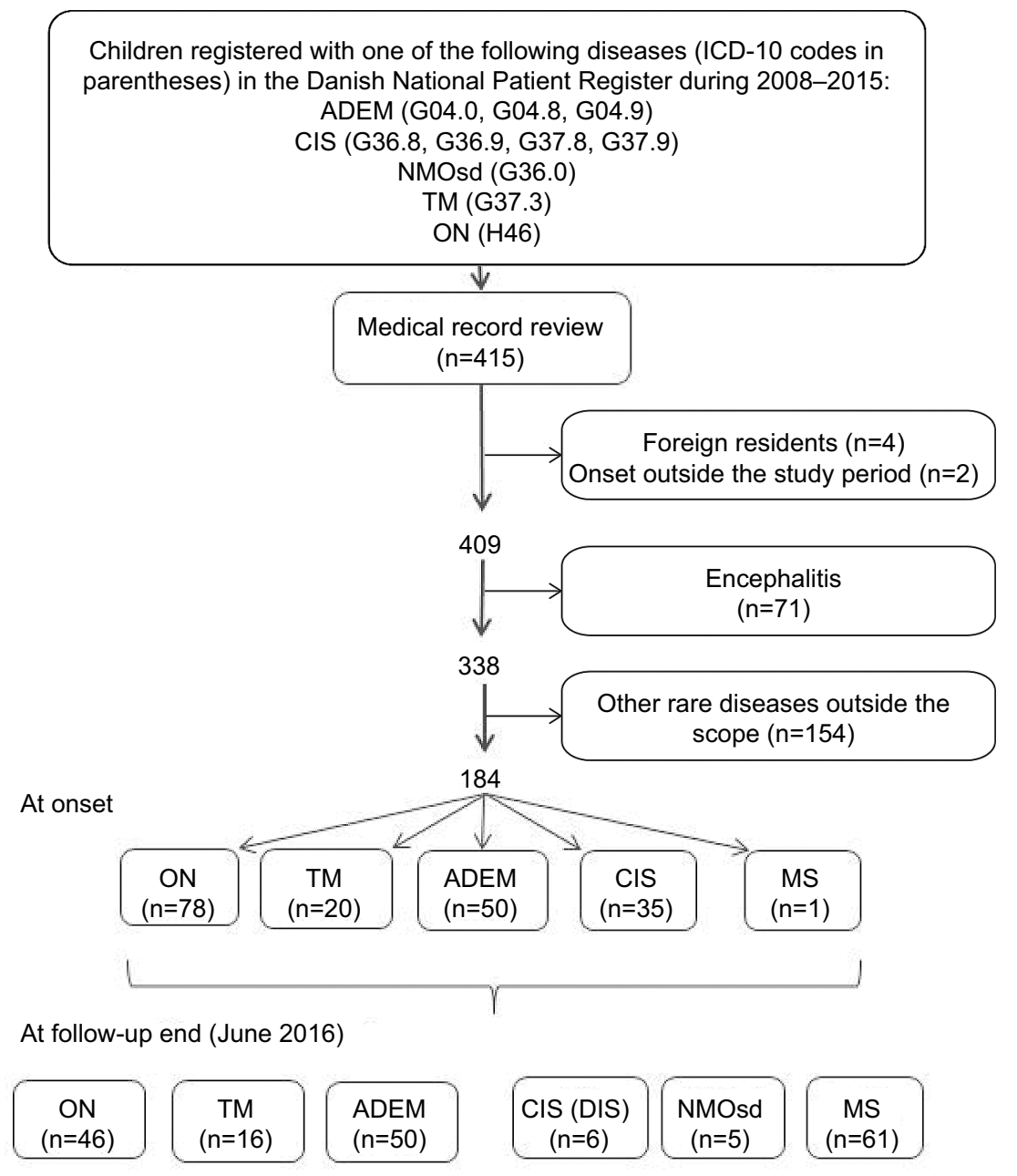

Figure I Flow diagram of the study population of children (<I8 years) with acquired demyelinating syndromes at onset and at follow-up end.

Abbreviations: ADEM, acute disseminated encephalomyelitis; CIS, clinically isolated syndrome; CIS (DIS), CIS including dissemination in space; ICD-I0, International Classification of Diseases revision 10; NMOsd, neuromyelitis optica spectrum disorder; ON, optic neuritis; TM, transverse myelitis. 
Table I Characteristics of all children in the study based on their validated diagnoses

\begin{tabular}{llll}
\hline ADS & $\begin{array}{l}\text { Number of } \\
\text { children }\end{array}$ & $\begin{array}{l}\text { Female, } \\
\mathbf{n}(\%)\end{array}$ & $\begin{array}{l}\text { Age at onset, } \\
\text { median (range) }\end{array}$ \\
\hline ADEM $^{\mathrm{a}}$ & 50 & $19(38)$ & $5.4(0.8-17.2)$ \\
$\mathrm{CIS}^{\mathrm{a}}$ & 35 & $25(71)$ & $15.9(3.4-17.9)$ \\
$\mathrm{ON}^{\mathrm{a}}$ & 78 & $54(69)$ & $16.3(4.1-18.0)$ \\
$\mathrm{TM}^{\mathrm{a}}$ & 20 & $7(35)$ & $12.6(2.5-17.6)$ \\
$\mathrm{NMOsd}^{\mathrm{b}}$ & 5 & $1(20)$ & $12.2(2.8-14.8)$ \\
$\mathrm{MS}^{\mathrm{b}}$ & $6 \mathrm{I}$ & $44(72)$ & $16.5(9.3-18.0)$ \\
$\mathrm{ADS}$ total & 184 & $116(58)$ & $14.8(0.8-18.0)$ \\
Children without & & & \\
validated ADS diagnosis & 225 & $110(49)$ & $9.1(0.0-17.9)$ \\
\hline
\end{tabular}

Notes: aDiagnosis at onset. Children with initially presumed monophasic ADS may progress to MS or NMOsd during follow-up. 'Diagnosis at the end of follow-up (June I, 2016). Children with MS or NMOsd may have initially been diagnosed with a monophasic ADS.

Abbreviations: ADEM, acute disseminated encephalomyelitis; ADS, acquired demyelinating syndrome; CIS, clinically isolated syndrome; MS, multiple sclerosis; NMOsd, neuromyelitis optica spectrum disorder; ON, optic neuritis; TM, transverse myelitis.

diagnosis was $0.71(95 \% \mathrm{CI}=0.62-0.80)$ for $\mathrm{ON}, 0.64(95 \%$ $\mathrm{CI}=0.43-0.82)$ for $\mathrm{TM}$, and $0.93(95 \% \mathrm{CI}=0.84-0.98)$ for MS (Table 2). The PPV was lower for CIS and ADEM $(<0.44)$, and due to few children with NMOsd, CIs were wide (PPV $=0.43 ; 95 \% \mathrm{CI}=0.10-0.82$ ). By restricting the analysis to having two or more diagnostic codes as either primary discharge diagnosis or secondary discharge diagnosis of the specific ADS, the PPV increased for ADEM, ON, TM, and NMOsd but considerably reduced the number of studied children. Interestingly, the inclusion of only primary diagnoses did not increase PPVs but only decreased the number of studied patients. When using any ADS as outcome (instead of the specific ADS), the PPVs increased markedly for CIS, TM, and NMOsd.

\section{ADEM and ICD- 10 codes}

As ADEM is the commonest ADS in children, we explored which diagnostic ICD-10 codes pediatricians usually used in children with a medical record verified diagnosis of ADEM. Among the 50 children who truly had ADEM, 29 children had at least one of the aforementioned ICD-10 codes of ADEM; among these children, 25 children had onset before the age of 10 years. However, among the remaining cohort of children without ADEM ( $n=359), 168$ (47\%) children also had an ICD-10 code of ADEM. Additionally, 94 children without ADEM had an ICD-10 code of ADEM and the code registration before the age of 10 years. The commonest nonADEM diseases coded by physicians using our prespecified ADEM codes were epilepsy and other forms of encephalitis.

\section{Progression from monophasic ADS to MS or NMOsd}

As the distinction between monophasic and relapsing diseases is important, we calculated the cumulative proportion of MS and NMOsd during follow-up in children initially diagnosed with presumed monophasic ADS (Table 3). During follow-up of 4.6 years (range $=0.3-9.4$ years), $33 \%$ of children initially diagnosed with presumed monophasic ADS progressed to MS. In the 118 children not progressing to MS or NMOsd, $97(82 \%)$ children were followed-up for $>2$ years and 111 (94\%) children were followed-up for $>1$ year. No children with

Table 2 PPVs of a validated ADS diagnosis for ICD-I0 codes in the Danish National Patient Register

\begin{tabular}{|c|c|c|c|c|c|c|c|c|}
\hline \multirow[t]{2}{*}{ Diagnosis } & \multicolumn{2}{|c|}{$\begin{array}{l}\text { Children with at least one } \\
\text { code as either primary } \\
\text { diagnosis or secondary } \\
\text { diagnosis (denominator) } \\
\text { and the specific medical- } \\
\text { record validated ADS } \\
\text { (numerator) }\end{array}$} & \multicolumn{2}{|c|}{$\begin{array}{l}\text { Children with at least two } \\
\text { codes as either primary } \\
\text { diagnosis or secondary } \\
\text { diagnosis (denominator) } \\
\text { and the specific medical- } \\
\text { record validated ADS } \\
\text { (numerator) }\end{array}$} & \multicolumn{2}{|c|}{$\begin{array}{l}\text { Children with at least } \\
\text { one code as primary } \\
\text { diagnosis (denominator) } \\
\text { and the specific medical- } \\
\text { record validated ADS } \\
\text { (numerator) }\end{array}$} & \multicolumn{2}{|c|}{$\begin{array}{l}\text { Children with at least } \\
\text { one code as either } \\
\text { primary diagnosis or } \\
\text { secondary diagnosis } \\
\text { (denominator) and any } \\
\text { medical-record validated } \\
\text { ADS (numerator) }\end{array}$} \\
\hline & $P^{d}$ & $\begin{array}{l}\text { PPV (exact } \\
95 \% \mathrm{Cl})\end{array}$ & $P^{d}$ & $\begin{array}{l}\text { PPV (exact } \\
95 \% \mathrm{CI})\end{array}$ & $P^{d}$ & $\begin{array}{l}\text { PPV (exact } \\
95 \% \mathrm{Cl})\end{array}$ & $P^{d}$ & $\begin{array}{l}\text { PPV (exact } \\
95 \% \mathrm{Cl})\end{array}$ \\
\hline ADEM & $29 / 197$ & $0.15(0.10-0.20)$ & $15 / 55$ & $0.27(0.16-0.4 I)$ & $25 / 148$ & $0.17(0.11-0.24)$ & $41 / 197$ & $0.20(0.15-0.27)$ \\
\hline $\mathrm{CIS}^{\mathrm{a}}$ & $31 / 117$ & $0.27(0.19-0.35)$ & $7 / 35$ & $0.20(0.8-0.37)$ & $29 / 103$ & $0.28(0.20-0.38)$ & $76 / 117$ & $0.65(0.56-0.74)$ \\
\hline ON & $77 / 108$ & $0.71(0.62-0.80)$ & $29 / 33$ & $0.88(0.72-0.97)$ & $58 / 81$ & $0.72(0.6 I-0.8 I)$ & $79 / 108$ & $0.73(0.64-0.81)$ \\
\hline TM & $16 / 25$ & $0.64(0.43-0.82)$ & $9 / 10$ & $0.90(0.56-1.00)$ & $14 / 22$ & $0.64(0.4 I-0.83)$ & $22 / 25$ & $0.88(0.69-0.97)$ \\
\hline NMOsd ${ }^{b}$ & $3 / 7$ & $0.43(0.10-0.82)$ & $2 / 2$ & $1.00(0.16-1.00)$ & $2 / 4$ & $0.50(0.07-0.93)$ & $5 / 7$ & $0.71(0.29-0.96)$ \\
\hline $\mathrm{MS}^{\mathrm{c}}$ & $55 / 59$ & $0.93(0.84-0.98)$ & $23 / 25$ & $0.92(0.74-0.99)$ & $48 / 51$ & $0.94(0.84-0.99)$ & $59 / 59$ & $1.00(0.93-1.00)$ \\
\hline All children & - & & - & & - & & $184 / 409$ & $0.45(0.40-0.50)$ \\
\hline
\end{tabular}

Notes: aDiagnosis at onset. 'Diagnosis at the end of follow-up. 'In the studied cohort, 59 children (in addition to non-MS diagnostic codes) were registered with a diagnostic code of MS (G35), of whom 55 truly had MS. The capture of MS was presumably incomplete for MS in Denmark during the same period. ${ }^{\mathrm{d}}$ The sum of the column numerators may exceed 184 because children can have both a monophasic ADS at onset and replasing disease at follow-up end. The sum of the column denominators may exceed 409 because children may be registered with more than one ADS code.

Abbreviations: ADEM, acute disseminated encephalomyelitis; ADS, acquired demyelinating syndrome; $\mathrm{Cl}$, confidence interval; CIS, clinically isolated syndrome; ICD-I0, International Classification of Diseases revision I0; MS, multiple sclerosis; NMOsd, neuromyelitis optica spectrum disorder; ON, optic neuritis; $P$, proportion; PPV, positive predictive value; TM, transverse myelitis. 
Table 3 Risk of developing MS or NMOsd during follow-up among children with an initial medical-record verified diagnosis of ADS

\begin{tabular}{|c|c|c|c|c|c|}
\hline \multirow[t]{3}{*}{ Diagnoses } & \multirow{3}{*}{$\begin{array}{l}\text { Follow-up (years), } \\
\text { mean } \pm \text { SD } \\
\text { (range) }\end{array}$} & \multicolumn{4}{|c|}{$\begin{array}{l}\text { Children initially diagnosed with monophasic ADS (denominator) } \\
\text { who developed MS or NMOsd during follow-up (numerator) }\end{array}$} \\
\hline & & \multicolumn{2}{|l|}{ MS } & \multicolumn{2}{|c|}{ NMOsd } \\
\hline & & $\mathbf{n}$ & Proportion (95\% CI) & $\mathbf{n}$ & Proportion $(95 \% \mathrm{Cl})$ \\
\hline ADEM & $4.6 \pm 2.3(0.8-8.3)$ & $0 / 50$ & $0(0.00-0.07)$ & $0 / 50$ & $0(0.00-0.07)$ \\
\hline $\mathrm{CIS}$ & $5.1 \pm 2.2(0.5-9.4)$ & $29 / 35$ & $0.83(0.66-0.93)$ & $0 / 35$ & $0(0.00-0.10)$ \\
\hline ON & $4.5 \pm 2.3(0.5-8.3)$ & $30 / 78$ & $0.38(0.28-0.50)$ & $2^{\mathrm{a} / 78}$ & $0.03(0.003-0.09)$ \\
\hline TM & $4.3 \pm 2.2(0.3-7.9)$ & $1 / 20$ & $0.05(0.00 \mathrm{I}-0.25)$ & $3^{\mathrm{b}} / 20$ & $0.15(0.03-0.38)$ \\
\hline All ADS & $4.6 \pm 2.3(0.3-9.4)$ & $6 I / / 84$ & $0.33(0.26-0.40)$ & $5 / 184$ & $0.03(0.009-0.06)$ \\
\hline
\end{tabular}

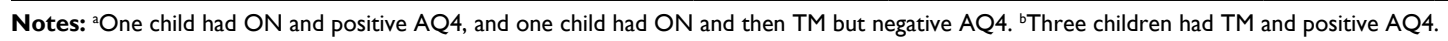

Abbreviations: AQ4, aquaporin-4 antibody; ADEM, acute disseminated encephalomyelitis; ADS, acquired demyelinating syndrome; Cl, confidence interval; CIS, clinically isolated syndrome; MS, multiple sclerosis; NMOsd, neuromyelitis optica spectrum disorder; ON, optic neuritis; SD, standard deviation; TM, transverse myelitis.

Table 4 Incidence rate, prevalence, sensitivity, specificity, positive and negative predictive values, and positive and negative likelihood ratios for each non-MS ADS group

\begin{tabular}{llllll}
\hline Outcome measure & ADEM $^{\mathbf{c}}$ & CIS (DIS) $^{\mathbf{d}}$ & NMOsd $^{\mathbf{d}}$ & ON $^{\mathbf{c}}$ & TM $^{\mathbf{c}}$ \\
\hline Incidence rate $^{\mathrm{a}}$ & 0.52 & 0.06 & 0.05 & $0.8 \mathrm{I}$ & 0.21 \\
Point prevalence $^{\mathrm{b}}$ & 9.35 & 0.72 & 0.70 & 2.93 & 1.76 \\
Sensitivity $(95 \% \mathrm{Cl})$ & $0.58(0.43-0.72)$ & $0.83(0.36-1.00)$ & $0.60(0.15-0.95)$ & $0.99(0.93-1.00)$ & $0.80(0.56-0.94)$ \\
Specificity $(95 \% \mathrm{Cl})$ & $1.00(1.00-1.00)$ & $1.00(1.00-1.00)$ & $1.00(1.00-1.00)$ & $1.00(1.00-1.00)$ & $1.00(1.00-1.00)$ \\
Positive predictive value $(95 \% \mathrm{Cl})$ & $0.15(0.10-0.20)$ & $0.04(0.05-0.17)$ & $0.43(0.10-0.82)$ & $0.71(0.62-0.80)$ & $0.64(0.43-0.82)$ \\
Negative predictive value $(95 \% \mathrm{Cl})$ & $1.00(1.00-1.00)$ & $1.00(1.00-1.00)$ & $1.00(1.00-1.00)$ & $1.00(1.00-1.00)$ & $1.00(1.00-1.00)$ \\
Positive likelihood ratio & 4,132 & 5,937 & 179,533 & 38,112 & 106,389 \\
Negative likelihood ratio & 0.42 & 0.17 & 0.40 & 0.01 & 0.20 \\
\hline
\end{tabular}

Notes: ${ }^{a} \mathrm{New}$ cases of chart-verified ADS per 100,000 person-years in children younger than 18 years. ${ }^{\mathrm{b}}$ The point prevalence in children (<18 years) was calculated as the sum of the age-specific incidence rates times the remaining years up to 18 years of age. 'Diagnosis at onset. 'Diagnosis at the end of follow-up.

Abbreviations: ADEM, acute disseminated encephalomyelitis; ADS, acquired demyelinating syndrome; Cl, confidence interval; CIS (DIS), clinically isolated syndrome including dissemination in space but not dissemination in time; MS, multiple sclerosis; NMOsd, neuromyelitis optica spectrum disorder; ON, optic neuritis; TM, transverse myelitis.

ADEM progressed to MS, NMOsd or multiphasic ADEM. A high risk for MS was seen in children initially diagnosed with ON (38\%) and CIS (83\%), but the MS risk was low in children with TM (5\%). In contrast, the highest risk for NMOsd was in children initially diagnosed with TM (15\%), whereas the risk of NMOsd was only $3 \%$ in children with ON.

\section{Other common outcome measures}

Assuming a complete coverage of pediatric non-MS ADS in Denmark during the study period, the incidence of ON was $0.81 / 100,000$ per year in children younger than 18 years and that of TM was $0.21 / 100,000$, but that of NMOsd was only $0.05 / 100,000$. The sensitivity was high for ON $(0.99 ; 95 \% \mathrm{CI}$ $=0.93-1.00)$ and $\mathrm{TM}(0.80 ; 95 \% \mathrm{CI}=0.56-0.94)$ but lower for $\operatorname{ADEM}(0.58 ; 95 \% \mathrm{CI}=0.43-0.72)$. For NMOsd $(0.60 ; 95 \%$ $\mathrm{CI}=0.15-0.95)$ and CIS (DIS) $(0.83 ; 95 \% \mathrm{CI}=0.36-1.00)$ the sensitivity CIs were too wide to make conclusions (Table 4). Due to the low prevalence of pediatric ADS, the PPVs were lower than the sensitivities. Specificities and negative predictive values were 1.00 for all non-MS ADS.

\section{Discussion}

Based on 415 children registered with at least one diagnostic ADS code in the NPR during 2008-2015 and using chart review as the gold standard for true diagnoses, we found an excellent PPV for MS $(0.93 ; 95 \% \mathrm{CI}=0.84-0.98)$ and acceptable PPV for ON $(0.71 ; 95 \% \mathrm{CI}=0.62-0.80)$ and $\mathrm{TM}(0.64$; 95\% CI $=0.43-0.82)$ but poor PPV for CIS $(0.27 ; 95 \%$ CI $=0.19-0.35)$ and $\operatorname{ADEM}(0.15 ; 95 \% \mathrm{CI}=0.10-0.20)$; NMOsd was inconclusive. Due to the low prevalence of pediatric ADS, the sensitivity was excellent for ON $(0.99 ; 95 \% \mathrm{CI}$ $=0.93-1.00)$ and fair for TM $(0.80 ; 95 \% \mathrm{CI}=0.56-0.94)$ but poor for $\operatorname{ADEM}(0.58 ; 95 \% \mathrm{CI}=0.43-0.72)$. During the mean follow-up of 4.6 years, $33 \%$ of children initially diagnosed with presumed monophasic ADS progressed to MS.

The strengths of our study are that it is population based and grounded in prospectively collected data from a nationwide administrative register with high completeness. In addition, all medical records $(\mathrm{n}=415)$ including follow-up case notes were reviewed by experienced pediatric neurologists. However, some limitations must be mentioned. The authors 
were not blinded to the children's ICD-10 codes during chart review. Furthermore, the MS cohort was not complete for the time period, and we were not able to have a secondary review of MRIs in the ADS cohort. The study design was retrospective, and consequently, more children with presumed monophasic ADS could have NMOsd with more systematical diagnostic workup. Finally, more children with monophasic ADS may progress to MS or NMOsd with longer follow-up.

Owing to the risk of developing a chronic demyelinating disease, children with non-MS ADS are referred to hospitals as either admissions or outpatient ambulatory consultations, with subsequent ICD-10 code notification to the NPR. For our cohort, only ICD-10 codes were used during the study period. We presume high completeness for ICD-10 codes because hospitals are reimbursed based on their reporting of ICD-10 codes in the Danish health care system, which is tax funded. Private consultant neurologists, pediatricians, or ophthalmologists always refer children to hospitals when suspecting demyelinating CNS disease; accordingly, it is unlikely that children with non-MS ADS are not registered with one of the aforementioned diagnostic codes. Our study for non-MS ADS is therefore nationwide and population based. Moreover, we accessed the medical records in all children who were registered with the ICD-10 codes during the study period. Furthermore, diagnostic criteria for ADS were relatively uniform for the study period.

We have previously estimated the annual incidence of pediatric MS as $0.88(95 \% \mathrm{CI}=0.71-1.09)$ per 100,000 person-years in children before the age of 18 years during 2008-2015, using the same data as for the current study but with additional information on incident MS cases from the Danish Multiple Sclerosis Registry. ${ }^{27-29}$ Therefore, our coverage of MS is $\sim 70 \%$. However, due to the natural reporting delay in MS, we may have underestimated the incidence of the disease because patients in our study period with their first neurological symptom may progress to MS with longer follow-up time. ${ }^{14,15}$ In addition, AQ4 is not routinely investigated in all children with TM or ON and some misclassification of children truly having NMOsd may have been present with more thorough diagnostic workup. Lack of uniformly accepted diagnostic criteria for ADEM may also complicate comparison with other studies because diagnostic criteria for true ADEM may differ. Follow-up of our cohort will presumably increase the proportion of children with monophasic ADS who progress to relapsing disease and thereby reduce this misclassification.

The PPV of ADEM (the commonest ADS in children) was low; therefore, we explored algorithms to improve PPV compared with the remaining children with non-ADEMADS, but as the PPV increased, the false positive rate increased considerably. One cause could be the lack of uniformly accepted diagnostic criteria for ADEM in Danish clinical practice. ${ }^{22}$ Furthermore, the physician giving the child an ICD-10 diagnostic code at discharge may be a general pediatrician not specialized in neurology. This may also be complicated by the existence of many ICD-10 codes for demyelinating diseases (illustrated by the prespecified diagnostic ICD-10 codes for ADEM) and lack of coding guidelines. The same applies to hemispheric demyelinating lesions causing hemisensory or motor deficits that physicians assume represent onset of MS (our CIS group).

We suggest that future ICD revisions create one category only for persons with brainstem or hemispheric syndrome of CIS and one for ADEM instead of multiple unspecific categories of acute demyelination. This should be accompanied by ICD coding guidelines for ADS to improve consistency in terminology. In addition, isolated ON or TM without cerebral demyelinating lesions should be coded differently from ON or TM with cerebral demyelinating lesions (CIS) due to the higher risk of disease progression to MS in the latter. Future research should focus on diagnostic ICD-10 coding of NMOsd in cohorts of children with ON and TM whose diagnostic workup includes the evaluation of AQ4 and MOG in all children.

\section{Conclusion}

PPV was high for MS and acceptable for ON and TM; these ICD-10 codes are therefore useful for epidemiological studies. In contrast, PPV was low for CIS and ADEM; NMOsd was inconclusive. Progression from monophasic ADS to MS/NMOsd, continual revisions and relaxation of diagnostic criteria of MS and NMOsd, varying diagnostic criteria for ADEM, and lack of ICD coding guidelines for ADS may decrease the validity of ADS diagnoses in the NPR.

\section{Acknowledgments}

We thank the chief physicians at Danish pediatric and neurological departments for their collaboration in accessing medical records. The study was supported by the Danish MS society (grant numbers A29625 and A31526), Novartis, Teva, and Genzyme.

\section{Author contributions}

All authors contributed toward data analysis, drafting and critically revising the paper, gave final approval of the version 
to be published, and agree to be accountable for all aspects of the work.

\section{Disclosure}

MSB has served on a scientific advisory board for Teva and has received speaker honoraria for lecturing from Novartis and support for congress participation from Teva, Novartis, and Roche. MM has served on a scientific advisory board for Biogen Idec, Novartis, and Merck Serono, has received honoraria for lecturing from Biogen Idec, Merck Serono, Novartis, and Genzyme, and has received support for congress participation from Biogen Idec, Novartis, Genzyme, and Teva. APB has received speaker honoraria from Novartis and has served on an advisory board for Biogen Idec. LCT has received honoraria for lecturing from Rigshospitalet (Copenhagen, Denmark), Statistics Denmark, and SanofiAvensis. This study was financially supported by the Danish MS Society, TEVA, Novartis and Genzyme. The authors report no other conflicts of interest in this work.

\section{References}

1. Lynge E, Sandegaard JL, Rebolj M. The Danish National Patient Register. Scand J Public Health. 2011;39(7 Suppl):30-33.

2. Andersen TF, Madsen M, Jørgensen J, Mellemkjoer L, Olsen JH. The Danish National Hospital Register. A valuable source of data for modern health sciences. Dan Med Bull. 1999;46(3):263-268.

3. Ben Ghezala I, Arendt JFB, Erichsen R, et al. Positive predictive value of the diagnosis coding for vitamin B12 deficiency anemia in the Danish National Patient Register. Clin Epidemiol. 2012;4:333-338.

4. Bork CS, Al-Zuhairi KS, Hansen SM, Delekta J, Joensen AM. Accuracy of angina pectoris and acute coronary syndrome in the Danish National Patient Register. Dan Med J. 2017;64(5):A5369.

5. Christensen J, Vestergaard M, Olsen J, Sidenius P. Validation of epilepsy diagnoses in the Danish National Hospital Register. Epilepsy Res. 2007;75(2-3):162-170.

6. Djurhuus BD, Skytthe A, Faber CE. Validation of the cholesteatoma diagnosis in the Danish National Hospital Register. Dan Med Bull. 2010;57(10):A4159.

7. Dydensborg Sander S, Størdal K, Plato Hansen T, et al. Validation of celiac disease diagnoses recorded in the Danish National Patient Register using duodenal biopsies, celiac disease-specific antibodies, and human leukocyte-antigen genotypes. Clin Epidemiol. 2016;8:789-799.

8. Hansen DL, Overgaard UM, Pedersen L, Frederiksen H. Positive predictive value of diagnosis coding for hemolytic anemias in the Danish National Patient Register. Clin Epidemiol. 2016;8:241-252.

9. Lasota AN, Overvad K, Eriksen HH, Tjønneland A, Schmidt EB, Grønholdt M-LM. Validity of peripheral arterial disease diagnoses in the Danish National Patient Registry. Eur J Vasc Endovasc Surg. 2017;53(5):679-685.

10. Lühdorf P, Overvad K, Schmidt EB, Johnsen SP, Bach FW. Predictive value of stroke discharge diagnoses in the Danish National Patient Register. Scand J Public Health. 2017;45(6):630-636.
11. Mason K, Thygesen LC, Stenager E, Brønnum-Hansen H, KochHenriksen N. Evaluating the use and limitations of the Danish National Patient Register in register-based research using an example of multiple sclerosis. Acta Neurol Scand. 2012;125(3):213-217.

12. Wermuth L, Lassen CF, Himmerslev L, Olsen J, Ritz B. Validation of hospital register-based diagnosis of Parkinson's disease. Dan Med J. 2012;59(3):A4391

13. Schmidt M, Pedersen L, Sørensen HT. The Danish Civil Registration System as a tool in epidemiology. Eur J Epidemiol. 2014;29(8):541-549.

14. Esbjerg S, Keiding N, Koch-Henriksen N. Reporting delay and corrected incidence of multiple sclerosis. Stat Med. 1999;18(13):1691-1706.

15. Thygesen LC, Keiding N, Koch-Henriksen N. Evaluating a parametric model to correct multiple sclerosis incidence for reporting delay. Stat Med. 2011;30(8):896-898.

16. Wingerchuk DM, Lennon VA, Pittock SJ, Lucchinetti CF, Weinshenker BG. Revised diagnostic criteria for neuromyelitis optica. Neurology. 2006;66(10):1485-1489.

17. Wingerchuk DM, Banwell B, Bennett JL, et al. International consensus diagnostic criteria for neuromyelitis optica spectrum disorders. Neurology. 2015;85(2):177-189.

18. McDonald WI, Compston A, Edan G, et al. Recommended diagnostic criteria for multiple sclerosis: guidelines from the International Panel on the diagnosis of multiple sclerosis. Ann Neurol. 2001;50(1):121-127.

19. Polman CH, Reingold SC, Edan G, et al. Diagnostic criteria for multiple sclerosis: 2005 revisions to the "McDonald Criteria." Ann Neurol. 2005;58(6):840-846.

20. Polman CH, Reingold SC, Banwell B, et al. Diagnostic criteria for multiple sclerosis: 2010 revisions to the McDonald criteria. Ann Neurol. 2011;69(2):292-302.

21. Krupp LB, Tardieu M, Amato MP, et al; International Pediatric Multiple Sclerosis Study Group. International Pediatric Multiple Sclerosis Study Group criteria for pediatric multiple sclerosis and immune-mediated central nervous system demyelinating disorders: revisions to the 2007 definitions. Mult Scler. 2013;19(10):1261-1267.

22. Boesen MS, Magyari M, Koch-Henriksen N, et al. Implications of the International Paediatric Multiple Sclerosis Study Group consensus criteria for paediatric acute disseminated encephalomyelitis: a nationwide validation study. Dev Med Child Neurol. In press 2018.

23. MikaeloffY, Caridade G, Husson B, Suissa S, Tardieu M, Neuropediatric KIDSEP Study Group of the French Neuropediatric Society. Acute disseminated encephalomyelitis cohort study: prognostic factors for relapse. Eur J Paediatr Neurol. 2007;11(2):90-95.

24. Benchimol EI, Manuel DG, To T, Griffiths AM, Rabeneck L, Guttmann A. Development and use of reporting guidelines for assessing the quality of validation studies of health administrative data. $J$ Clin Epidemiol. 2011;64(8):821-829.

25. Pedersen CB. The Danish Civil Registration System. Scand J Public Health. 2011;39(7 Suppl):22-25.

26. Statistikbanken [Stat Bank]. Seneste Opdateringer [Latest updates]; cited October 31, 2016. Available from: http://www.statistikbanken.dk/ statbank5a/default.asp?w=1920. Accessed November 1, 2017. Danish.

27. Boesen MS, Magyari M, Koch-Henriksen N, et al. Pediatric-onset multiple sclerosis and other acquired demyelinating syndromes of the central nervous system in Denmark during 1977-2015: a nationwide population-based incidence study. Mult Scler. Epub 2017 Jun 1.

28. Koch-Henriksen N, Rasmussen S, Stenager E, Madsen M. The Danish Multiple Sclerosis Registry. History, data collection and validity. Dan Med Bull. 2001;48(2):91-94.

29. Koch-Henriksen N, Magyari M, Laursen B. Registers of multiple sclerosis in Denmark. Acta Neurol Scand Suppl. 2015;132(199):4-10. 


\section{Supplementary materials}

Table SI ICD-I 0 diagnostic groups, codes, and their description

\begin{tabular}{lll}
\hline Diagnostic groups & ICD-I0 codes & Description \\
\hline Multiple sclerosis & G35 & Multiple sclerosis \\
Neuromyelitis optica spectrum disorders & G36.0 & Neuromyelitis optica (Devic) \\
Acute disseminated encephalomyelitis & G04.0 & Acute disseminated encephalitis \\
& G04.8 & Other encephalitis, myelitis, and encephalomyelitis \\
Transverse myelitis & G04.9 & Encephalitis, myelitis, and encephalomyelitis, unspecified \\
Optic neuritis & G37.3 & Acute transverse myelitis in demyelinating disease of the central nervous system \\
Clinically isolated syndrome & $\mathrm{H} 46$ & Optic neuritis \\
& $\mathrm{G} 36.8$ & Other specified acute disseminated demyelination \\
& $\mathrm{G} 36.9$ & Acute disseminated demyelination, unspecified \\
& $\mathrm{G} 37.8$ & Other specified demyelinating diseases of the central nervous system
\end{tabular}

Abbreviation: ICD-I0, International Classification of Diseases revision 10.

Clinical Epidemiology

\section{Publish your work in this journal}

Clinical Epidemiology is an international, peer-reviewed, open access, online journal focusing on disease and drug epidemiology, identification of risk factors and screening procedures to develop optimal preventative initiatives and programs. Specific topics include: diagnosis, prognosis, treatment, screening, prevention, risk factor modification,

Submit your manuscript here: https://www.dovepress.com/clinical-epidemiology-journal

\section{Dovepress}

systematic reviews, risk and safety of medical interventions, epidemiology and biostatistical methods, and evaluation of guidelines, translational medicine, health policies and economic evaluations. The manuscript management system is completely online and includes a very quick and fair peer-review system, which is all easy to use. 